OPEN

SUBJECT AREAS:

SURFACE

SPECTROSCOPY

SCANNING PROBE

MICROSCOPY

MATERIALS SCIENCE

MICROSCOPY

Received

26 June 2014

Accepted

2 October 2014

Published

24 October 2014

Correspondence and requests for materials should be addressed to J.N. (nakamura@ims. tsukuba.ac.jp)

* These authors contributed equally to this work.

\title{
Principles and Application of Heterodyne Scanning Tunnelling Spectroscopy
}

\author{
Eiji Matsuyama*, Takahiro Kondo*, Haruhiro Oigawa, Donghui Guo, Shojiro Nemoto \& Junji Nakamura
}

Faculty of Pure and Applied Sciences, University of Tsukuba, 1-1-1 Tennodai, Tsukuba, Ibaraki, 305-8573 Japan.

Detection of the extremely weak signals in spectroscopy over an extremely wide frequency region is central to diverse sciences, including materials science, biology, astronomy and chemistry. Here we show a new type of atomic-scale spectroscopy, heterodyne scanning tunnelling spectroscopy (HSTS), which is based on the innovative application of the nonlinear heterodyne-mixing detection at the metal-insulator-metal (MIM) heterojunction of STM tip-vacuum-sample. The principle of HSTS is identical to that of the Atacama Large Millimeter Array (ALMA) space telescope in terms of using heterojunction for detecting extremely weak signals by converting from terahertz region to lower frequency regions. The MIM detector of ALMA, which is composed of niobium-titanium-nitride ( $\mathrm{NbTiN}$ ) tip-insulator- $\mathrm{NbTiN}$, is very similar in shape and size to that of HSTS. We successfully detect a heterodyne beat signal $f_{3}\left(=\left|f_{2}-f_{1}\right|\right)$ and intermodulation distortion via tunnelling current by superimposing two different AC signals, $f_{1}$ and $f_{2}$, onto the DC tunnelling current at a highly oriented pyrolytic graphite (HOPG) surface. We then obtain spectra of the localized electronic states of HOPG by using $f_{3}$. HSTS can be performed with a high resolution and over a wide energy range, including the terahertz range.

\footnotetext{
canning tunnelling microscopy (STM) has been extensively used to perform atomic-scale spectroscopy on surface-quantised energy levels, such as vibrational and spin modes ${ }^{1-5}$. However, the detection of very weak signals of a single atom at the thermal noise limit, such as a single spin, or the collection of its fine-structured spectrum is still difficult ${ }^{6}$. This difficulty is ascribed to perturbation of the quantised energy levels by the excitation source and to poor energy resolution and/or low sensitivity of the detectors used in scanning tunnelling spectroscopy (STS). To overcome these difficulties, here we show a new type of atomic-scale spectroscopy, heterodyne scanning tunnelling spectroscopy (HSTS), which is based on the innovative application of the nonlinear heterodyne-mixing detection at the metal-insulator-metal (MIM) heterojunction of STM tip-vacuum-sample ${ }^{7,8}$. The principle of HSTS is identical to that of the Atacama Large Millimeter Array (ALMA) space telescope in terms of using heterojunction for detecting extremely weak signals by converting from terahertz region to lower frequency regions. The MIM detector of ALMA, which is composed of niobium-titanium-nitride (NbTiN) tip-insulator-NbTiN, is very similar in shape and size to that of HSTS $^{9-11}$.
}

\section{Results}

In scanning tunnelling microscopy (STM) measurements, the STM tip is located close (0.4-0.7 nm) to the sample surface to achieve tunnelling conditions ${ }^{12}$. The tunnelling junction is thus created at the tip-vacuum-sample region, which is regarded as a tiny metal-insulator-metal junction. The tunnelling currents thus exhibit a nonlinear dependence on the sample bias, as shown in Fig. 1a. We first generated and detected the heterodyne mixing signal in the STM measurements of highly oriented pyrolytic graphite (HOPG). A two-tone AC signal was superimposed onto a DC tunnelling current. The two-tone signal consists of the sinusoidal wave $\left(f_{2}\right)$ and the fundamental component $\left(f_{1}\right)$ of the distorted rectangular wave (see Methods). Frequency $f_{1}$ was maintained at $3000 \mathrm{~Hz}$, whereas frequency $f_{2}$ was varied as $3750,3500,3250,2750,2500$ and $2250 \mathrm{~Hz}$. We then measured the AC components in the tunnelling current using a fast Fourier transform (FFT). As shown in Figs. 1b and 1c, differential signals of $f_{3}=\left|f_{2}-f_{1}\right|$ were clearly observed in addition to the $f_{1}$ and $f_{2}$ signals, indicating that the $f_{3}$ signal was the heterodyne differential beat signal; i.e., the heterodyne mixing occurred because of the so-called 'squared law'. Thus frequency $f_{3}$ can be intentionally and finely tuned using heterodyne mixing. Note that we could generate and detect $f_{3}$ signal with audio frequency of $\mathrm{kHz}(3.623 \mathrm{kHz})$ in tunnelling current by the heterodyne mixing of two microwave $f_{1}(1.999996380 \mathrm{GHz})$ and $f_{2}(2.000000020 \mathrm{GHz})$ signals (see Supplementary Information).

We subsequently controlled the intensity of the beat signal of $f_{3}$ by changing the intensity of $f_{2}$ while maintaining a constant intensity of $f_{1}$. Fig. 2 clearly indicates that the intensities of $f_{3}$ increased linearly with increasing 

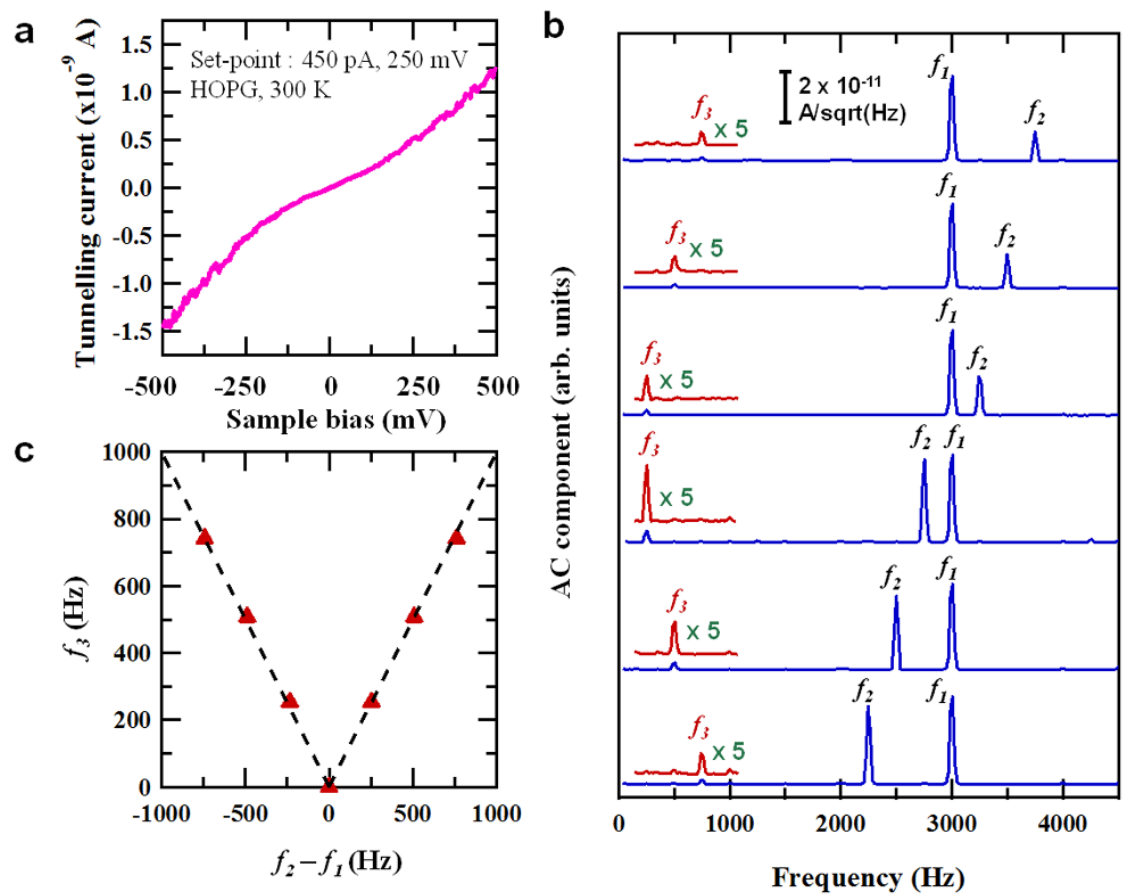

Figure $1 \mid$ Generation and detection of the heterodyne beat signal. (a), $I-V$ curves: Tunnelling current as a function of the sample bias of HOPG. (b), Spectral densities of $f_{1}, f_{2}$ and $f_{3}$ with variation of the frequency of $f_{2}$ (blue curves). The spectra at the $f_{3}$ region are shown with fivefold intensity by red curves. The FFT resolution was set as $19.53 \mathrm{~Hz}$. (c), The $f_{3}$ frequency plotted against the $f_{2}-f_{1}$ frequency. In all cases, measurements were conducted at $300 \mathrm{~K}$ (set-point: tunnelling current $I_{\mathrm{t}}=450 \mathrm{pA}$ and sample bias $V_{\mathrm{s}}=250 \mathrm{mV}$ ).

intensity of $f_{2}$ and that the intensity of $f_{3}$ was always one order of magnitude smaller than that of $f_{2}$ because of a heterodyne loss. The deviation from linearity is attributed to the thermal drift of the STM instrument at $300 \mathrm{~K}$. The intensity of the beat signal can thus be well controlled through input signals.

Notably, side-band signals are generated when we superimposed a two-tone signal with differential frequency of $10 \mathrm{~Hz}\left(f_{1}=610.0\right.$ and $f_{2}=600.0 \mathrm{~Hz}$ ) as shown in Fig. 3 (see Supplementary Information). The side-band signals are observed at the lower and upper side of the two-tone signal. The upper-side-band peaks are also observed at 30$80 \mathrm{~Hz}$. These side-band signals are ascribed to so-called 'intermodulation distortion (IMD)', i.e., the side-band signals are generated by the interaction between the two AC signals with similar frequencies in the two-tone signal at a non-linear junction. Important point is
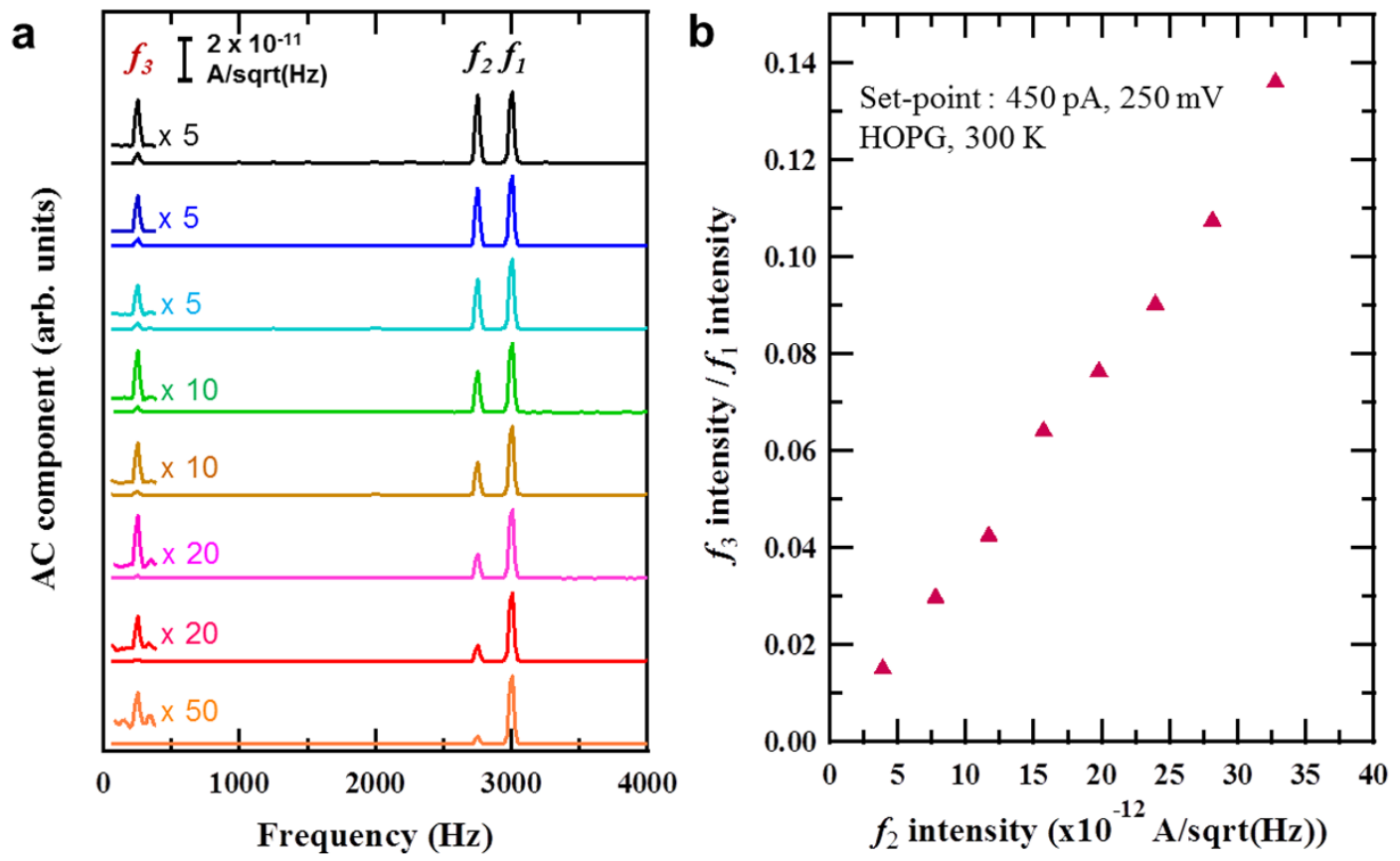

Figure $2 \mid$ Intensity control of the heterodyne beat signal. (a), Spectral density of $f_{1}(3007.8 \mathrm{~Hz}), f_{2}(2753.9 \mathrm{~Hz})$ and $f_{3}(253.9 \mathrm{~Hz})$ with variation of the intensity of $f_{2}$ at $300 \mathrm{~K}$ (set-point: tunnelling current $I_{\mathrm{t}}=450 \mathrm{pA}$ and sample bias $V_{\mathrm{s}}=250 \mathrm{mV}$ ). The FFT resolution was set as $19.53 \mathrm{~Hz}$. The spectra at the $f_{3}$ region are also shown with $5(10,20$ or 50$)$ times greater intensity in each spectrum. (b), $\left(f_{3}\right.$ intensity/ $f_{1}$ intensity) plotted as a function of the $f_{2}$ intensity. 


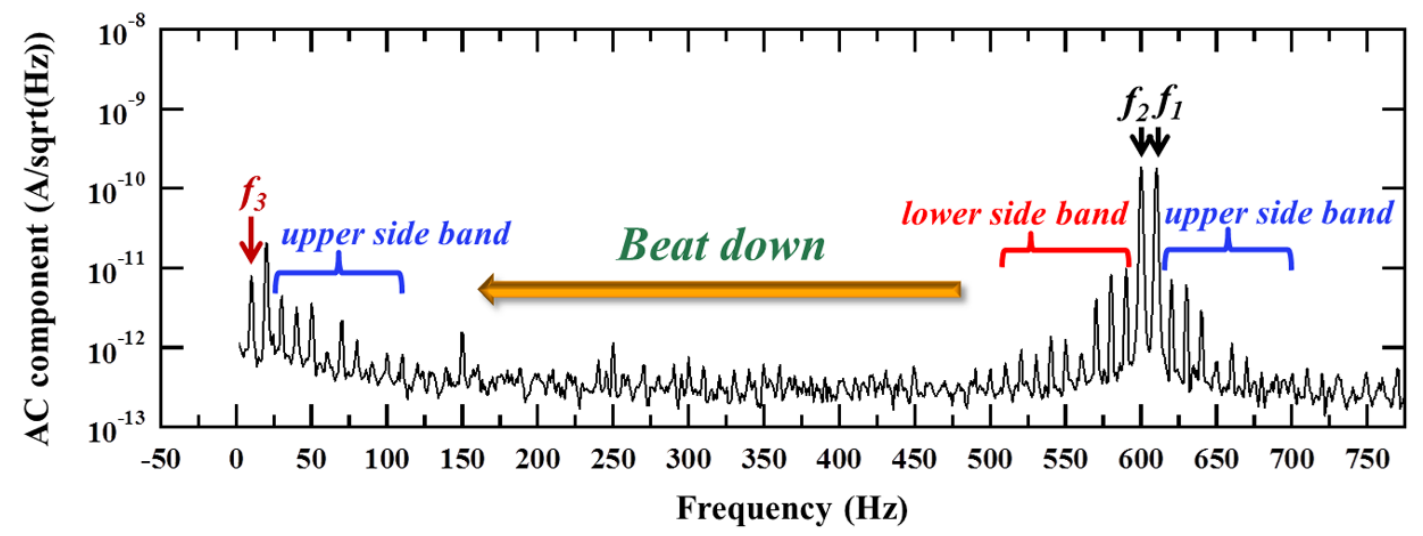

Figure 3 Intermodulation distortion. Spectral density of AC components in tunnelling current when the two-tone signal comprising two AC signals, $f_{1}$ and $f_{2}\left(f_{1}=610.0\right.$ and $f_{2}=600.0 \mathrm{~Hz}$ ), are superimposed onto the DC tunnelling current at $4.0 \mathrm{~K}$ (set-point: tunnelling current $I_{\mathrm{t}}=350 \mathrm{pA}$ and sample bias $V_{\mathrm{s}}=75 \mathrm{mV}$ ). The FFT resolution was set as $9.77 \mathrm{~Hz}$.

that the IMD has been observed at the tunnelling junction of STM for the first time.

On the basis of these results, we propose a new concept of spectroscopy: heterodyne scanning tunnelling spectroscopy (HSTS), as shown in Fig. 4 . The concept is to use the $f_{3}$ heterodyne beat signal as an excitation source as well as a probe of quantised states. Because the heterodyne mixing occurs at the tunnelling junction, the spectroscopy can be performed at the selected position only and with atomic resolution $^{13}$. The intensity of the $f_{3}$ signal is sufficiently weak to avoid unnecessary perturbation of quantised states, such as local heating of the sample. Various types of HSTS are expected. For example, resonance/absorption spectroscopy can be performed by scanning the frequency of the heterodyne beat signal, $f_{3}$, when the two-tone signal comprising two AC signals, $f_{1}$ and $f_{2}$, is input into tunnelling currents. The intensity of $f_{3}$ is simultaneously measured to perform the resonance/absorption spectroscopy. In another method, quantised states with very weak signals with a thermal noise limit as low as $-174 \mathrm{dBm}^{14}$ can be regarded as $f_{2}$. In this case, the $f_{1}$ AC signal with a sufficiently large intensity (such as an intensity of $0 \mathrm{dBm}$ ) is input, and the $f_{3}$ heterodyne beat signal is detected as a probe signal. The surface quantised states with $f_{2}$ can thus be effectively identified with amplification of heterodyne detection. Furthermore, the $f_{3}$ beat signal can be used as a probe in conventional STS measurements, as described later. HSTS offers three unique characteristics:

\section{i) Selective energy region}

The frequency of the $f_{3}$ signal can be very precisely controlled in the megahertz-to-petahertz $(\mathrm{MHz}-\mathrm{PHz})$ frequency range by controlling the input, $f_{1}$ and $f_{2}$, AC signals using coherent radiation, such as microwave and ultraviolet radiations. Thus, HSTS can be used to perform local spectroscopic measurements in the terahertz $(\mathrm{THz})$ frequency region, which is currently difficult with other techniques because useful power generation and receiver technologies are inefficient and impractical (this problem is thus referred to as the ' $\mathrm{THz}$ gap' $)^{9,15}$. In principle, HSTS can access an extremely wide energy range, from the nano-electron volt $(\mathrm{neV})$ to the electron volt $(\mathrm{eV})$,

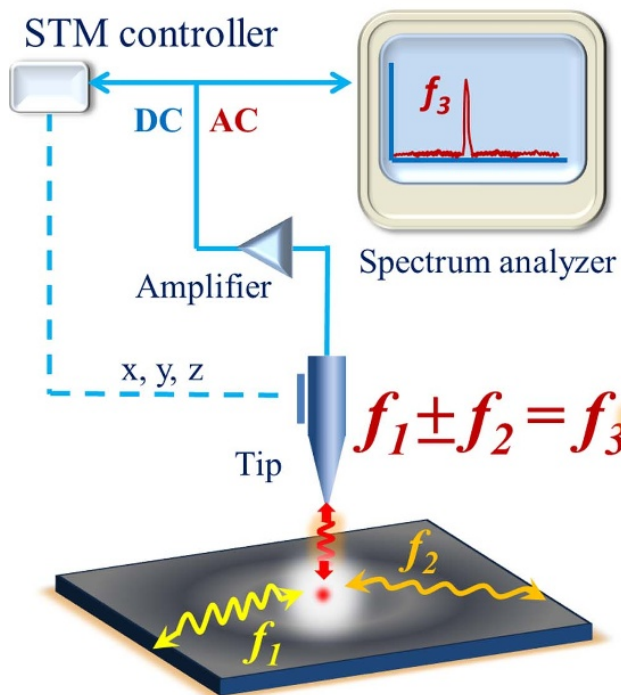

Input-tunable $f_{1}$ and $f_{2}$ signals

\section{Selected energy region}

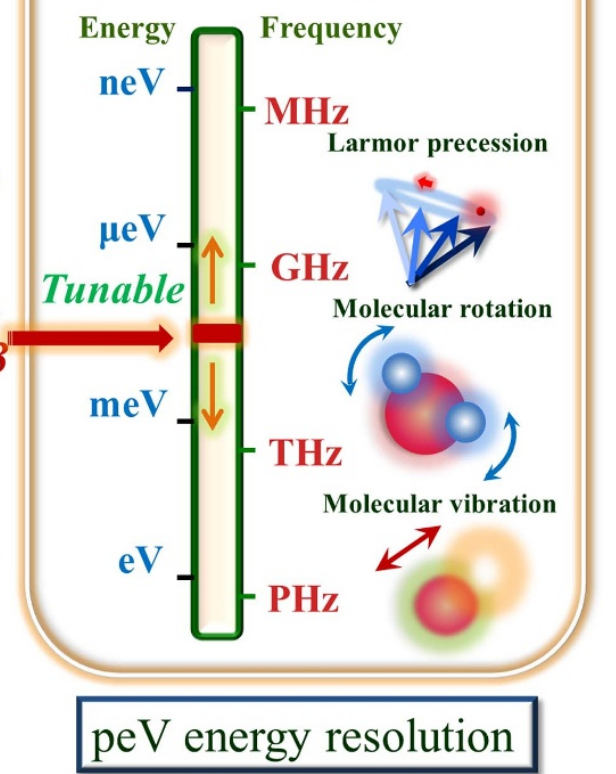

Figure $4 \mid$ Schematic of heterodyne scanning tunnelling spectroscopy (HSTS). HSTS can access a wide energy range from the nano-electron volt (neV) to the electron volt $(\mathrm{eV})$ range to perform fine spectroscopy with pico-electron volt (peV) energy resolution and atomic spatial resolution (see text). 

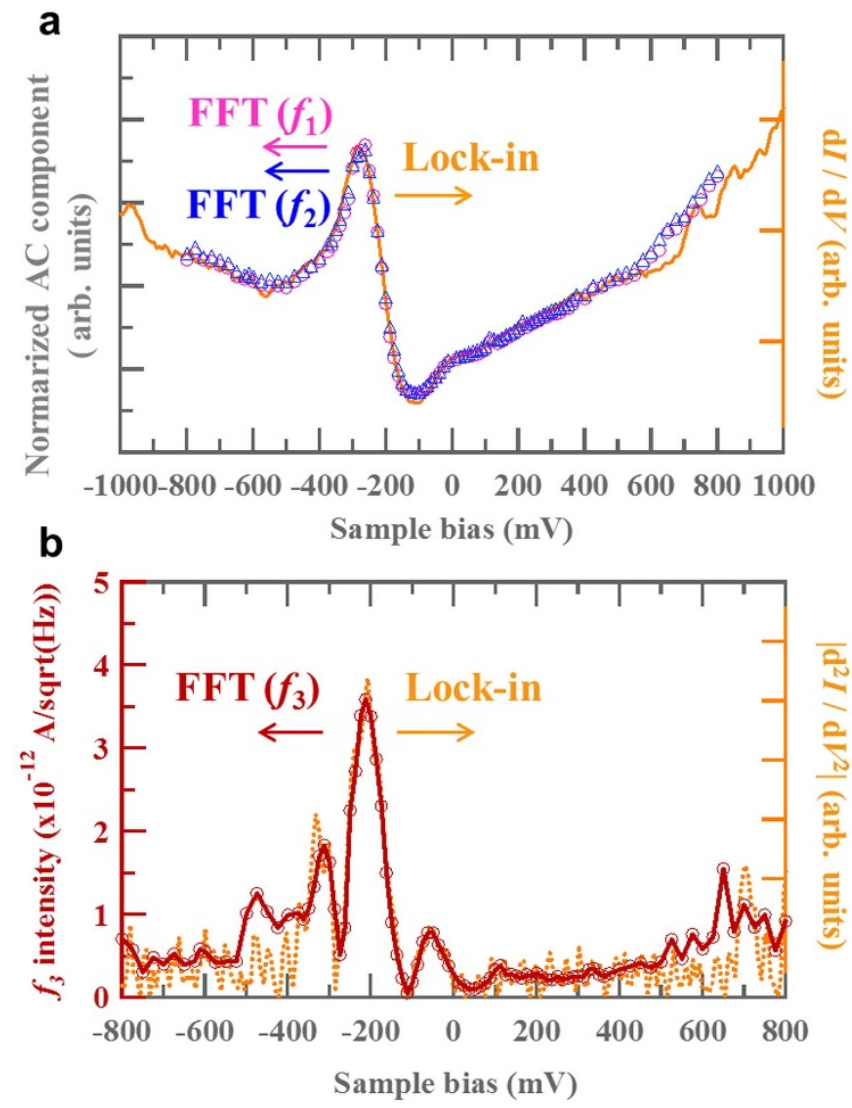

Figure 5 Example of the application of HSTS. (a), Conventional STS spectrum measured by the lock-in detection method (solid line) and HSTS spectra measured by FFT (circle and triangle plots for the $f_{1}$ and $f_{2}$ signals, respectively). The measurements were conducted at the same position near the defect of HOPG at $3.23 \mathrm{~K}$ (set-points in both conventional STS and HSTS: tunnelling current $I_{t}=100 \mathrm{pA}$ and sample bias $V_{s}=150 \mathrm{mV}$ ). For HSTS, the intensities of $f_{1}(3501.0 \mathrm{~Hz})$ and $f_{2}(3200.7 \mathrm{~Hz})$ were measured by FFT after normalization by the intensity of input signals of $f_{1}$ and $f_{2}$ as functions of the sample bias (see Supplementary Information). The FFT resolution was set as $2.44 \mathrm{~Hz}$. The conventional STS spectrum was measured using the lock-in detection technique with a modulation bias of $20 \mathrm{mV}$ (peak-to-peak amplitude) at $3.5 \mathrm{kHz}$. (b), The intensity of the heterodyne beat signal $f_{3}(300.3 \mathrm{~Hz})$ measured by FFT was plotted as a function of the sample bias (red circles). The absolute value of the numerically conducted first derivative of the $\mathrm{d} I / \mathrm{d} V$ spectrum measured by the lock-in detection technique $\left(\left|\mathrm{d}^{2} I / \mathrm{d} V^{2}\right|\right.$, dashed line $)$ is plotted on the right $y$-axis.

for fine spectroscopy. HSTS can be used to construct a twodimensional map of the selected specific energy level with atomic spatial resolution.

\section{ii) High energy resolution and time resolution}

Energy resolution of $f_{3}$ on the order of pico-electron volts (peV) can be attained by locking the input signals, $f_{1}$ and $f_{2}$, to the external reference signals with high accuracy, such as those of quartz, rubidium and caesium standards ${ }^{10,16,17}$. For example, Larmor precession of the single spin (gigahertz, GHz) in a sample or molecular rotation/ vibration of an adsorbed molecule (THz) on a sample can be investigated at peV resolution with atomic spatial resolution, as suggested in Fig. 4. Time-resolved spectroscopy, such as investigating the dynamical reaction behaviour of atoms and molecules or spin dynamics $^{18-20}$, can also be analysed by HSTS because of the FFT relationship between 'time domain' and 'frequency domain' ${ }^{21}$. For example, at the resonant condition, the phase-change dynamics under the specific external field of the specific energy mode can be analysed by detecting the intensity as a function of the input phase of $f_{1}$.

iii) Fine structure analysis

In general, the analysis of fine structures in electron spin resonance (ESR) or infrared absorption (IR) is very important because they contain rich information of the quantised states and their interactions. In HSTS, we observed that IMD generates the side-bands shown in Fig. 3, which correspond to the fine structure in the spectra. The fine structure can thus be analysed by beat down in a lowerfrequency region.

Finally, we demonstrate the application of HSTS and compare the results with those obtained by conventional STS measured by the lock-in detection method. Fig. 5a shows the spectrum obtained for conventional STS using the lock-in detection method (solid line) and spectra obtained for HSTS (circle and triangle plots). Both conventional STS and HSTS measurements were conducted at the same tip position near the defect of HOPG with the same tip-sample distance at 3.23 K. For HSTS measurements, we superimposed the two-tone signal comprising two $\mathrm{AC}$ signals of $f_{1}$ $(3501.0 \mathrm{~Hz})$ and $f_{2}(3200.7 \mathrm{~Hz})$ onto the DC tunnelling current. The AC components of $f_{1}$ and $f_{2}$ and the heterodyne beat signal of $f_{3}$ in the tunnelling current were measured by FFT; the intensities of these components are plotted in Figs. 5a and 5b after normalization by the intensity of the input AC signals (see Supplementary Information). The agreement between the results of conventional STS and HSTS in Fig. 5a indicates that the $f_{1}$ and $f_{2}$ intensities are proportional to the first derivative of the tunnelling current, $\mathrm{d} I / \mathrm{d} V$. Here, in addition to the parabolic shape of the spectrum due to the tails of the $\pi$ and $\pi^{*}$ bands of graphite, a localised state peak near the Fermi level was observed at $-285 \mathrm{mV}$. This peak is considered to represent the localised states related to the non-bonding $\mathrm{p}_{\mathrm{z}}$ orbital (edge state) of carbon because of the partial breaking of the $\pi$-conjugated system of the graphite surface by the presence of the defect ${ }^{22-24}$. With respect to the $f_{3}$ signal, the $f_{3}$ intensity is clearly proportional to the second derivative of the tunnelling current, $\mathrm{d}^{2} I / \mathrm{d} V^{2}$, as shown in Fig. 5b. These results can be explained as follows:

The tunnelling current $I\left\{E+k\left(\sin \omega_{1} \mathrm{t}+\sin \omega_{2} \mathrm{t}\right)\right\}$ can be represented as

$$
\begin{aligned}
& I\left\{E+k\left(\sin \omega_{1} \mathrm{t}+\sin \omega_{2} \mathrm{t}\right)\right\} \\
& =I(E)+I^{\prime}(E) k\left(\sin \omega_{1} \mathrm{t}+\sin \omega_{2} \mathrm{t}\right)+\frac{1}{2} I^{\prime \prime}(E) k^{2} \\
& \left\{1+\cos \left(\omega_{1}-\omega_{2}\right) t-\cos \left(\omega_{1}+\omega_{2}\right) t-\frac{1}{2} \cos \left(2 \omega_{1} \mathrm{t}\right)-\frac{1}{2} \cos \left(2 \omega_{2} \mathrm{t}\right)\right\}^{(1)} \\
& +\ldots
\end{aligned}
$$

where $I(E)$ is the DC tunnelling current at sample bias $E$ and $k\left(\sin \omega_{1} \mathrm{t}\right)$ and $k\left(\sin \omega_{2} \mathrm{t}\right)$ are the applied AC biases. The intensities of both AC signal components with frequencies $\omega_{1}$ and $\omega_{2}$ are proportional to the first derivative of the tunnelling current, $I^{\prime}(E)$, which corresponds to the local density of states in STS ${ }^{12}$. Thus, the plots of $f_{1}\left(=\omega_{1} / 2 \pi\right)$ and $f_{2}\left(=\omega_{2} / 2 \pi\right)$ exhibit the same shape as the conventional STS spectrum as shown in Fig. 5a. In equation (1), the intensity of the heterodyne differential component $\left(\omega_{1}-\omega_{2}\right)$ is proportional to the second derivative of the tunnelling current, $I^{\prime \prime}(E)$. Thus, the plots of $f_{3}\left(=\left|\omega_{1}-\omega_{2}\right| / 2 \pi\right)$ exhibit the same shape as the derivative of the STS spectrum $\left(\mathrm{d}^{2} I / \mathrm{d} V^{2}\right)$ as presented in Fig. $5 \mathrm{~b}$. These results indicate that HSTS has been established such that the tunnelling junction simultaneously produces the heterodyne beat signal (second derivative of the tunnelling current) and homodyne signal (first derivative of the tunnelling current). Thus, HSTS can also be applied to the inelastic electron tunnelling spectroscopy (IETS) measurement ${ }^{12}$. 


\section{Discussion}

In this work, we applied the heterodyne mixing method used in the ALMA space telescope to STM with the heterojunction of the STM tip-vacuum-sample. The heterodyne beat signals were observed to be generated by two different high-frequency signals via sample bias in the STM measurement of HOPG. Then, we successfully obtained the STS spectra using the heterodyne mixing method. The detection of the heterodyne signal is expected to open a new class of STS for the detection of any quantum energy level of a single atom with high resolution. In principle, the HSTS can access any energy level-from the nano-electron volt to the electron volt-with high-precision pico-electron volt energy resolution without unnecessary perturbation of the measurement system.

\section{Methods}

Supplementary Fig. S1 shows a schematic of our experimental setup. All measurements were performed in a UHV STM chamber (USM-1400, UNISOKU) with a base pressure of $1 \times 10^{-8} \mathrm{~Pa}$. A commercial STM controller (SPM controller, Nanonis) and a Pt-Ir tip (STM probe, Pt:Ir $=8: 2$, UNISOKU) were used. Fresh HOPG (ZYAgrade, Panasonic) was used as the sample; it was cleaved in air using adhesive tape and then placed in the UHV chamber. Two different frequency AC signals (i.e., a two-tone signal) were applied to the sample surface under a DC sample bias voltage in the STM system. One of the AC signals was generated by the STM controller (this signal exhibited a distorted rectangular waveform, where the fundamental wave is used as one of the signals in the two-tone signal) and the other AC sinusoidal signal was applied to the sample by an external oscillator (HP 3324A synthesised function/sweep generator, Agilent). Heterodyne beat signals were detected via the tunnelling current. The tunnelling current was amplified by a pre-amp (DLPCA200, FEMT), and then detected by a pico-ammeter integrated into the STM controller. The spectral density of the tunnelling current was obtained via a spectrum analyser integrated into the STM controller; FFT with a resolution of $19.5,9.77$ or $2.44 \mathrm{~Hz}$ was performed. In this work, each FFT spectrum was obtained without data accumulation. The frequency region for HSTS measurements was selected as the $\mathrm{kHz}$ region to verify the HSTS model theory, at which the effects of floating capacitances, inductances and resistances on the spectra ${ }^{14}$ are negligible. The comb signal that should be originated from the distorted rectangular wave will be described in our future publication. Here, we discuss the heterodyne mixing between two input AC signals of two sinusoidal waves (the fundamental wave of distorted rectangular wave and the sinusoidal wave).

1. Zhang, R. et al., Chemical mapping of a single molecule by plasmon-enhanced Raman scattering. Nature 498, 82-86 (2013).

2. Guo, D. et al., Observation of Landau levels in potassium-intercalated graphite under a zero magnetic field. Nat Commun. 3, 1068 (2012).

3. Loth, S. et al., Controlling the state of quantum spins with electric currents. Nat Phys. 6, 340-344 (2010).

4. Heinrich, A. J., Gupta, J. A., Lutz, C. P. \& Eigler, D. M. Single-Atom Spin-Flip Spectroscopy. Science 306, 466-469 (2004).

5. Stipe, B. C., Rezaei, M. A. \& Ho, W. Single-Molecule Vibrational Spectroscopy and Microscopy. Science 280, 1732-1735 (1998).

6. Balatsky, A. V., Fransson, J., Mozyrsky, D. \& Manassen, Y. STM NMR and nuclear spin noise. Phys. Rev. B 73, 184429 (2006).

7. Kemiktarak, U., Ndukum, T., Schwab, K. C. \& Ekinci, K. L. Radio-frequency scanning tunnelling microscopy. Nature 450, 85-88 (2007).

8. Stranick, S. J. \& Weiss, P. S. Alternating Current Scanning Tunneling Microscopy and Nonlinear Spectroscopy. J. Phys. Chem. 98, 1762-1764 (1994).

9. Tonouchi, M. Cutting-edge terahertz technology. Nat Photon. 1, 97-105 (2007).
10. Musha, M. et al., A highly stable mm-wave synthesizer realized by mixing two lasers locked to an optical frequency comb generator. Opt. Comm. 240, 201-208 (2004).

11. Atacama Large Millimeter/submillimeter Array (ALMA), Available at: http:// www.almaobservatory.org/ [Date of Access: 10/06/2014].

12. Wiesendanger, R. Ed., Scanning probe microscopy and spectroscopy: methods and applications. (Cambridge University Press, 1994).

13. Völcker, M., Krieger, W. \& Walther, H. Laser-driven scanning tunneling microscope. Phys. Rev. Lett. 66, 1717-1720 (1991).

14. Messina, P. et al. Spin noise fluctuations from paramagnetic molecular adsorbates on surfaces. J. Appl. Phys. 101, 053916 (2007).

15. Siegel, P. H. Terahertz Technology. IEEE Transactions Microwave Theory Tech. 50, 910-928 (2002)

16. Kourogi, M., Nakagawa, K. \& Ohtsu, M. Wide-span optical frequency comb generator for accurate optical frequency difference measurement. IEEE Journal of Quantum Electronics 29, 2693-2701 (1993).

17. Akamatsu, D. et al., Spectroscopy and frequency measurement of the $87 \mathrm{Sr}$ clock transition by laser linewidth transfer using an optical frequency comb. Appl. Phys. Exp. 7, 012401 (2014).

18. Cocker, T. L. et al., An ultrafast terahertz scanning tunnelling microscope. Nat Photon 7, 620-625 (2013).

19. Loth, S. et al., Measurement of Fast Electron Spin Relaxation Times with Atomic Resolution. Science 329, 1628-1630 (2010).

20. Koppens, F. H. L. et al., Driven coherent oscillations of a single electron spin in a quantum dot. Nature 442, 766-771 (2006).

21. Cundiff, S. T. \& Ye, J. Colloquium: Femtosecond optical frequency combs. Rev. Mod. Phys. 75, 325-342 (2003).

22. Fujita, M., Wakabayashi, K., Nakada, K. \& Kusakabe, K. Peculiar Localized State at Zigzag Graphite Edge. J. Phys. Soc. Jpn. 65, 1920-1923 (1996).

23. Niimi, Y. et al., M. \& Fukuyama, H. Scanning tunneling microscopy and spectroscopy studies of graphite edges. Applied Surf. Sci. 241, 43-46 (2005).

24. Kondo, T. et al., Edge states propagating from a defect of graphite: Scanning tunneling spectroscopy measurements. Phys. Rev. B 82, 153414 (2010).

\section{Acknowledgments}

This work was supported by JSPS KAKENHI, Grant Numbers 21920021. This work was also supported by the New Energy and Industrial Technology Development Organization.

\section{Author Contributions}

E.M. proposed original idea of HSTS. J.N. supervised the project. E.M., T.K. and D.G. carried out the experiments. All the authors (E.M., T.K., H.O., D.G., S.N. and J.N.) discussed the results and E.M., T.K., H.O. and J.N. wrote the manuscript.

\section{Additional information}

Supplementary Information accompanies this paper at http://www.nature.com/ scientificreports

Competing financial interests: The authors declare no competing financial interests. How to cite this article: Matsuyama, E. et al. Principles and Application of Heterodyne Scanning Tunnelling Spectroscopy. Sci. Rep. 4, 6711; DOI:10.1038/srep06711 (2014).

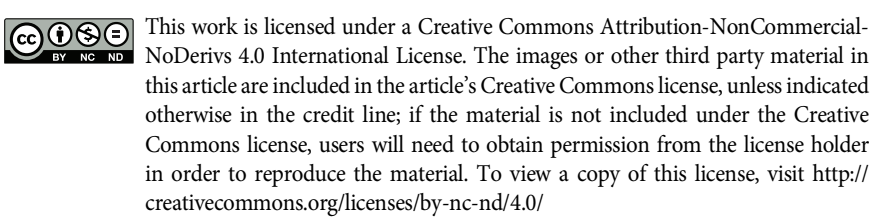

